\title{
JAK2 Inhibition: Reviewing a New Therapeutical Option in Myeloproliferative Neoplasms
}

\author{
Mar Bellido and Peter A. W. te Boekhorst \\ Department of Hematology, Erasmus University Medical Center, P.O. Box 2040, 3000 CA Rotterdam, The Netherlands \\ Correspondence should be addressed to Peter A. W. te Boekhorst, p.teboekhorst@erasmusmc.nl
}

Received 12 September 2011; Revised 29 November 2011; Accepted 4 December 2011

Academic Editor: Mark R. Litzow

Copyright ( $) 2012$ M. Bellido and P. A. W. te Boekhorst. This is an open access article distributed under the Creative Commons Attribution License, which permits unrestricted use, distribution, and reproduction in any medium, provided the original work is properly cited.

\begin{abstract}
$J A K 2$ is a tyrosine kinase gene that plays an essential role in the development of normal haematopoiesis. Hyperactivation of JAK2 occurs in myeloproliferative neoplasms by different mechanisms. As a consequence, JAK2 inhibitors have been designed to suppress the cytokine signalling cascade caused by the constitutive activation of JAK2. In clinical trials, JAK2 inhibitors are efficient in decreasing spleen size, controlling clinical symptoms, and improving quality of life in patients with myeloproliferative neoplasms. However, JAK2 inhibitors are unable to target uncommitted hematopoietic progenitors responsible of the initiation of the myeloproliferative disease. It is expected that, in order to cure the myeloproliferative disease, JAK2 inhibitors should be combined with other drugs to target simultaneously different pathways and to target the initiator hematopoietic cell population in myeloproliferative disorders. Taking advantage of the inhibition of the cytokine cascade of JAK2 inhibitors, these compounds are going to be used not only to treat patients with hematological neoplasms but may also be beneficial to treat patients with rheumatoid arthritis or other inflammatory diseases.
\end{abstract}

\section{Introduction}

Myeloproliferative neoplasms (MPNs) are clonal disorders up to now characterized by the autonomous proliferation of committed hematopoietic progenitors secondary to an aberrant activation of tyrosine kinase (TK) signalling pathways in combination with an exaggerated response to hematopoietic cytokines and growth factors $[1,2]$. Constitutive activation of TKs is a consistent molecular signature in cell proliferation. Examples of Constitutive activation of TKs are seeing in solid tumours [3,4], rheumatoid arthritis [5], and hematopoietic malignancies [6]. Known mechanisms of TK activation may result from acquired heterozygote of homozygote point mutations $[7,8]$, internal tandem duplications [9], and chromosomal translocations $[10,11]$. The knowledge of the molecular mechanism involved in the pathogenesis of chronic myeloid leukemia (CML) [12] has allowed to elucidate the molecular dissection of chronic proliferation in MPN. Using CML as paradigm of constitutive activation of TK in chronic myeloproliferation, James et al. sequenced the coding exons and intron-exon junctions of JAK2 in 3 polycythemia vera
(PV) patients and 2 controls. In 2 of these patients a G-to-T mutation at nucleotide 1849 in exon 12 was found, leading to a substitution of valine to phenylalanine at position 617 (V617F). This mutation was not a polymorphism, but a recurrent acquired mutation that was found in granulocytes, erythroblasts, and platelets of 40 out of 45 PV patients but not in any controls or patients with secondary erythrocytosis [13]. JAK2V617F was also found in other BCR-ABL negative MPN [14]. JAK2V617F occurs in the pseudokinase domain of the JAK2 gene. The mutated pseudokinase domain is not able to negatively regulate the kinase domain of JAK2, resulting in an autonomous activation of the JAK2 kinase domain with subsequently persistent phosphorylation of STAT and MAPK proteins $[15,16]$ and hyperstimulation of the cytokine signalling pathway [17]. As a consequence, cells expressing the JAK2V617F mutation are hypersensitive to hematopoietic cytokine stimulation, resulting in an abnormal erythroid-, myeloid-, and thromboproliferation. Moreover, JAK2-deficient mice do not survive because of absence of erythropoiesis. Myeloid progenitors of these mice fail to respond to EPO, GM-CSF, and thrombopoietin stimulation 
[18]. These experiments demonstrate that JAK2 plays an essential role in the development of normal hematopoiesis.

Not all patients with "classical" MPN carry the JAK2V6$17 \mathrm{~F}$ mutation. This mutation is present in almost all PV patients, but only in half of the patients with essential thrombocythemia (ET) and primary myelofibrosis (PMF), suggesting that there are alternative routes of $J A K$-cytokine signalling activation [14]. In the most prevalent $B C R-A B L 1$ negative MPN (PV, ET, and PMF), up to now it is known that $J A K 2$ activation may also occur by JAK2 mutations in exon 12 [19], by mutations in the inhibitory adaptor protein LNK [20], or by a TEL-JAK2 chromosomal translocation that induces erythropoietin-independent erythroid differentiation and myelofibrosis [21]. Other mutations described in MPN but also in other haematological malignancies are mutations in the thrombopoietin receptor at codon 515 (MPL) [22], the isocitrate dehydrogenase family genes $(I D H)$, the additional sex combs like 1 gene (ASXL1), the casitas B-lineage lymphoma proto-oncogene $(C B L)$, the TET oncogene family member 2 (TET2), and the Ikaros family zinc finger 1 gene (IKZF1) [23]. These mutations promote hematopoietic proliferation via other pathways than $J A K$-cytokine signalling activation and they have been detected more frequently in MPN other than PV, ET of PMF, myelodysplastic syndromes, secondary acute myeloid leukemia, and blast-transformation phase of MPN.

There is a relationship between the underlying genetic mechanism of the MPN and the response to different treatments. For example, $40-50 \%$ of the patients with primary PMF and ET who carried JAK2V617F mutation had decreases in proportion of JAK2-mutated DNA [24]. About $20 \%$ of the PMF and ET patients who carried MPL mutations had no decreases in proportion of $M P L$-mutated DNA when treated with JAK2 inhibitors but they did have it when treated with biological response modifiers (pegylatedinterferon alpha 2a, lenalidomide, and prednisone) [24].

New biological properties of JAK2 are currently being investigated. Recently, it has been demonstrated that JAK2 is not only localised in the cytoplasm of hematopoietic cells where JAK2 plays a role in the cytokine signalling pathway, but also in the nucleus of hematopoietic cells [25]. In the nucleus, a hyperactive JAK2 induces phosphorylation of the histone $\mathrm{H} 3$ at tyrosine residue 41 (H3Y41), releasing the transcriptional repressor heterochromatin protein lalfa (HP1a) from chromatin and resulting in an activation of transcription of genes repressed by HP1a, as the oncogene LMO2. LMO2 might play a role in the pathogenicity of MPN. It is remarkable that H3Y41 phosphorylation requires a hyperactive JAK2, implying that the activation of different pathways with different JAK2 effectors and different target genes is involved in the pathogenesis of MPN.

In the era of JAK2 inhibitors, this finding opens new possibilities for combined therapeutic targets (JAK2 inhibitors and histone posttranslational modificators) that may benefit patients with MPN [26].

\section{Current Therapies for $B C R-A B L 1$ Negative MPN (PV, ET, and PMF) Based on JAK2 Inhibitors and Their Combinations}

Hyper-activation of JAK2 is a critical step in the pathogenesis of $B C R-A B L 1$ negative, classical MPN.

Autonomous activation of the JAK2 kinase domain with subsequently persisting phosphorylation of STAT and MAPK proteins occurs in patients with and without JAK2V617F mutations. JAK2 inhibitors have been designed to suppress the cytokine signalling induced by a hyperactive cytoplasmatic JAK2 gene. JAK2 inhibitors compete for the ATP-binding pocket of the tyrosine kinase domain of JAK2 [27]. Because JAK2V617F mutation is localized outside the ATP-binding site, JAK2 inhibitors do not discriminate between JAK2 and $J A K 2$-mutated genes [28]. In consequence, JAK2 inhibitors can be used in patients with MPN independently of the JAK2 mutation status.

Nowadays, several JAK2 inhibitors are tested in clinical trials in Europe and USA (INCB018424, TG101348, CYT387, CEP-701, AZD1480, SB1518, and XL019) and others (NCBO16562, NVP-BSK805, and R723) are in preclinical development.

INCB018424 known as Ruxolitinib is a potent and selective JAK1 and JAK2 inhibitor. It has been used in patients with PMF, where INCB018424 downregulates proinflammatory cytokines through the inhibition of JAK1 and suppresses the phosphorylated signal of STAT3 through the inhibition of $J A K 2$, independently of the presence of JAK2V617F mutation [29].

In animal models of JAK2V617F mutation MPN, oral INCB018424 markedly reduced splenomegaly and circulating levels of inflammatory cytokines and preferentially eliminated neoplastic cells, resulting in significantly prolonged survival without myelosuppressive or immunosuppressive effects [30].

In patients with PMF treated with INCB018424, a significant reduction of constitutional symptoms and more than $50 \%$ reduction of splenomegaly occurred. Clinical benefits were associated with a marked decrease of levels of circulating inflammatory cytokines, although the load of JAK2V6$17 \mathrm{~F}$ was marginally reduced [29]. Myelosuppression grade 3 or 4 was observed in less than $10 \%$ of the patients.

TG101348, also known as SAR302503, is a selective smallmolecule JAK2 antagonist that inhibits primary hematopoietic cells derived from patients with MPN and with JAK2V617F, MPLW515K, JAK2 exon 12 mutations as well as mutation negative patients [31]. In animal models of JAK2V617F positive MPN, TG101348 reduced erythrocyte and leukocyte counts, the extramedullary hematopoiesis, and myelofibrosis without toxicities [31, 32]. Biologically, TG101348 reduced the JAK2V617F disease burden, and it was demonstrated a suppression of endogenous erythroid colony formation and inhibition of phosphorylated STAT5 [31]. In patients with myelofibrosis, TG101348 induced a reduction of the spleen size according to the criteria of International Working Group for Myelofibrosis Research and Treatment (IWG-MRT) [33] and a normalization of blood counts after 6 and 12 cycles 
[34]. A significant decrease in JAK2V617F allele burden was observed at 6 months in mutation-positive patients with a consistent decrease at 12 months [34].

CYT387 is an ATP-competitive potent JAK1, JAK2, and tyrosine kinase 2 (TYK2) inhibitor, at nanomolar concentrations, more selective against JAK2 than against other tyrosine kinases in comparison with other JAK2 inhibitors [35]. Its effect has been analyzed in cell lines harboring mutated JAK2 alleles, showing an inhibition of growth of erythroid colonies and erythroleukemia cells and inhibition of STAT5 phosphorylation.

In a murine MPN model, CYT387 normalized erythrocytes, leukocytes, spleen size, and restored physiologic levels of inflammatory cytokines. Biologically, there was a reduction of the JAK2V617F allele burden. However, after cessing therapy, JAK2V617F positive cells persisted and MPN relapsed [35].

CEP-701 known as Lestaurtinib is an Fms-like tyrosine kinase 3 (FLT3) inhibitor in current use on acute myeloid leukemia clinical trials and a JAK2 kinase inhibitor which suppresses phosphorylation induced by JAK2 tyrosine kinase. In patients with PV, CEP-701 inhibited growth of expanded erythroid cells [36]. In 22 patients with PMF who carried out the JAK2V617F mutation, CEP-701 induced a modest clinical recovery with mainly improvement of the spleen size. Biologically there was no improvement in bone marrow fibrosis or JAK2V617F allele burden [37]. Mainly toxicities have a high incidence of any grade of gastrointestinal toxicity in $9-72 \%$ of the patients and haematological toxicity grade 3-4 in $14-23 \%$ of the patients [37].

$J A K 2$ inhibitors can be compared with $B C R-A B L 1$ inhibitors, since both sort of drugs are TK inhibitors. Nevertheless, whereas $B C R-A B L 1$ inhibitors are directed against an aberrant fusion gene [38], JAK2 inhibitors are directed against a gene which is present in normal cells and have an important role in the development of normal hematopoiesis. This means that adverse events are induced with JAK2 inhibitors at doses to be able to control the myeloproliferative phenotype, inducing often grade 3-4 hematological toxicity as seen in clinical trials, limiting the clinical efficiency of JAK2 inhibitors. Different studies describe the incidence of reversible grade 3 or 4 hematological toxicity between $3-35 \%$ depending on the inhibitor specificity $[34,37,39]$. Other common adverse events are gastrointestinal symptoms, probably related to the inhibition of other tyrosine kinases. The incidence of nausea, vomiting, and diarrhoea varies between $5-70 \%$ depending on the compound [34, 37, 39].

Up to now it is known that JAK2 is a member of a family of tyrosine kinases present in the cytoplasm of hematopoietic cells. Recently, it has been demonstrated that JAK2 is also present in the nucleus of hematopoietic cells where it indirectly activates the expression of oncogenes as LMO2 [25]. It is not yet well known whether JAK2 inhibitors have a role in the inhibition of the JAK2 nuclear function. In the next years, the increasing clinical and biological experience with $J A K 2$ inhibitors will clarify their role.

Although imatinib treatment in CML cannot directly be compared with JAK2 inhibition in MPN, it can be used as a model of clinical experience with TK inhibitors. Therefore, we can speculate about what it is going to happen with the use of JAK2 inhibitors in the clinical practice. One might expect the drug resistance to JAK2 inhibitors by acquisition of mutations in the ATP-binding pocket of the TK domain of JAK2 and/or via the amplification of JAK2. We can also expect that JAK2 inhibitors are efficient to alleviate the clinical symptoms of patients with MPN, when used as monotherapy, but inefficient to cure the disease as happened with imatinib [40]. Clinical trials using JAK2 inhibitors for patients with myelofibrosis have demonstrated the effectiveness of these drugs to reduce clinical symptoms splenomegaly and to improve quality of life. However, in these trials, JAK2 burden was slightly reduced, indicating that JAK2 inhibitors are efficient to block the cytokine pathway responsible of the clinical symptoms in patients with MPN, but are not enough efficient to block the main molecular mechanism that originates the disease. Recently, Mullally et al. [41], using a JAK2V617F knock-in mouse MPN model, have shown that JAK2 inhibitors are able to control the hyperproliferation of committed hematopoietic progenitors in MPN, but are unable to eliminate the cell population from which the initiator clone arises. This cell population has been identified as noncommitted hematopoietic progenitors JAK2V617F+, Lin-, Sca+ c-kit+ (LSK positive). JAK2 inhibitors may have to be used in combination with drugs which target the LSK positive noncommitted population to be able to cure MPN.

Given the fact that JAK2 inhibitors induce myelosuppression but cannot cure MPN, combinations with other compounds that might have therapeutic synergy with JAK2 inhibitors seem to be mandatory. In this sense, interferon-alpha therapy is a good option to be associated to JAK2 inhibitors, due to its multiple effects on the regulation of immune modulatory cells, the expression of apoptotic genes, inhibition of angiogenesis, suppression of the proliferation of hematopoietic progenitor cells, and promoting the cycling of hematopoietic stem cells $[42,43]$. It is thought that interferon-alpha can also inhibit the cytokine signalling coming from bone marrow stromal cells to support proliferation and survival of malignant cells in MPN. Recently, Manshouri et al. have demonstrated that humoral factors secreted by the bone marrow stromal cells protect malignant cells carrying $J A K 2 \mathrm{~V} 617 \mathrm{~F}$ from the therapeutic effect of the JAk2 inhibitors [44]. Thus, combination of JAK2 inhibitors and interferon alpha could be a more efficient therapeutic regimen to treat MPN patients than only JAK2 inhibitors.

Other immunomodulatory drugs are also been tested in MPN patients, mainly in those with myelofibrosis. Thalidomide and lenalidomide with or without prednisone have shown efficacy to inhibit the increased cytokine production in these patients, decreasing the spleen size, myelofibrosis, and inhibiting angiogenesis [45]. Pomalidomide, another analogue, is currently being evaluated with or without prednisone in large clinical trials to treat patients with myelofibrosis [46]. These immunomodulatory drugs are candidates to be associated to JAK2 inhibitors as targeting therapy in patients with MPN.

Classical therapies, as hydroxycarbamide, are also effective to treat patients with MPN, not only as cytoreduction therapy but also as therapy to decrease the JAK2V617F load. 
TABLE 1: Current therapies for BCR-ABL1 negative MPN patients to be combined with JAK2 inhibitors.

\begin{tabular}{|c|c|c|c|c|}
\hline Drug & Target & Results & JAK2V617F load & Reference \\
\hline \multicolumn{5}{|l|}{ JAK2 inhibitors } \\
\hline $\begin{array}{l}\text { INCB018424 } \\
\text { Ruxolitinib }\end{array}$ & $J A K 2, J A K 1$ & $\begin{array}{l}>50 \% \text { reduction in } \\
\text { splenomegaly and } \\
\text { constitutional symptoms }\end{array}$ & $\begin{array}{l}\text { JAK2V617F load } \\
\text { marginally reduced }\end{array}$ & Verstovsek et al. [29] \\
\hline $\begin{array}{l}\text { TG101348 or } \\
\text { SAR302503 }\end{array}$ & JAK2, FLT3 & Reduction in splenomegaly & $\begin{array}{l}\text { JAK2V617F load } \\
\text { significantly reduced }\end{array}$ & Pardanani et al. [34] \\
\hline CYT387 & JAK2, JAK1, TYK2 & $\begin{array}{l}\text { In a murine model, } \\
\text { normalized erythrocytes, } \\
\text { leukocytes, spleen size, and } \\
\text { levels of inflammatory } \\
\text { cytokines }\end{array}$ & $\begin{array}{l}J A K 2 \mathrm{~V} 617 \mathrm{~F} \text { load } \\
\text { reduced }\end{array}$ & Tyner et al. [35] \\
\hline $\begin{array}{l}\text { CEP-701 } \\
\text { Lestaurtinib }\end{array}$ & JAK2, FLT3 & Reduction in splenomegaly & $\begin{array}{l}J A K 2 \mathrm{~V} 617 \mathrm{~F} \text { load no } \\
\text { reduced }\end{array}$ & $\begin{array}{l}\text { Hexner et al. [36] } \\
\text { Santos et al. [37] }\end{array}$ \\
\hline \multicolumn{5}{|l|}{ Immunomodulators } \\
\hline Interferon-alpha & Stromal cells & $\begin{array}{l}\text { Inhibit cytokine signalling, } \\
\text { proliferation and } \\
\text { angiogenesis }\end{array}$ & Unknown & Kiladjian et al. [43] \\
\hline Thalidomide & Stromal cells & Inhibition of angiogenesis & Unknown & Jabbour et al. [45] \\
\hline Lenalidomide & Stromal cells & Inhibition of angiogenesis & Unknown & Jabbour et al. [45] \\
\hline Pomalidomide & Stromal cells & Inhibition of angiogenesis & Unknown & Begna et al. [46] \\
\hline \multicolumn{5}{|l|}{ Other } \\
\hline Hydroxycarbamide & $J A K 2$ mutant burden & Inhibition of proliferation & $\begin{array}{l}\text { JAK2V617F load } \\
\text { significantly reduced }\end{array}$ & Besses et al. [47] \\
\hline $\begin{array}{l}\text { Histone } \\
\text { post-translationa } \\
\text { modificators }\end{array}$ & $J A K 2$ nuclear & $\begin{array}{l}\text { Depletion of JAK2V617F } \\
\text { autophosphorylation, } \\
\text { apoptosis in vitro }\end{array}$ & Unknown & Wang et al. [26] \\
\hline
\end{tabular}

Recently, Besses et al. have shown that hydroxycarbamide can reduce the JAK2 mutant load to more than $50 \%$ in untreated patients diagnosed with PV and TE [47]. This effect has synergy with the therapeutic effect of JAK2 inhibitors, making hydroxycarbamide a candidate therapy to be combined with $J A K 2$ inhibitors.

$J A K 2$ inhibitors are effective to alleviate clinical symptoms in patients with $B C R-A B L$ negative MPN. Combination with other therapies which show synergy and other biological properties than JAK2 inhibitors is promising as the most effective therapy in these disorders (Table 1).

\section{Final Remarks}

JAK2 is a tyrosine kinase gene which plays an essential role in the development of normal haematopoiesis and in the cytokine signalling pathway involving STAT3 and STAT5 proteins. Hyperactivation of JAK2 occurs in MPN by different genetic mechanisms. JAK2 inhibitors have been designed to suppress the cytokine cascade initiated by the activation of $J A K 2$, independently of the underlying genetic mechanism. In phase II/III of clinical trials, JAK2 inhibitors are efficient to control clinical symptoms and to improve quality of life. Recently, it has been demonstrated that JAK2 can also be located in the nucleus of cells, where it plays a role in the transcriptional control of genes regulated by HP1a. Whether $J A K 2$ inhibitors may have a role in the suppression of the nuclear role of $J A K 2$ or not is at the moment unknown, but it opens new insights for the combined use of epigenetic therapy with JAK2 inhibitors for the treatment of MPN.

Recently, it has also been demonstrated that JAK2 inhibitors are unable to target uncommitted hematopoietic progenitors responsible of the initiation of the myeloproliferative disease. It is expected that, in order to cure the MPN disease, JAK2 inhibitors should be combined with other drugs to target simultaneously different pathways and to target the initiator hematopoietic LSK positive cell population in MPN.

Taking advantage of the inhibition of the cytokine cascade of JAK2 inhibitors, these compounds are going to be used not only to treat patients with MPN, but also patients with autoimmune diseases as rheumatoid arthritis, Crohn's disease, or Colitis ulcerosa.

\section{Authors' Contribution}

M. Bellido and P. Boekhorst conceived the study, P. Boekhorst reviewed the paper and contributed to the final draft. M. Bellido declares that there are no competing financial interests in relation to this work. P. Boekhorst is a member of an advisory board of Novartis.

\section{References}

[1] M. Bennett and D. F. Stroncek, "Recent advances in the bcr-abl negative chronic myeloproliferative diseases," Journal of Translational Medicine, vol. 4, article 41, 2006. 
[2] A. Stoddart, M. L. Dykstra, B. K. Brown, W. Song, S. K. Pierce, and F. M. Brodsky, "Lipid rafts unite signaling cascades with clathrin to regulate BCR internalization," Immunity, vol. 17, no. 4, pp. 451-462, 2002.

[3] I. Duran, S. J. Hotté, H. Hirte et al., "Phase I targeted combination trial of sorafenib and erlotinib in patients with advanced solid tumors," Clinical Cancer Research, vol. 13, no. 16, pp. 4849-4857, 2007.

[4] M. C. Heinrich, H. Joensuu, G. D. Demetri et al., "Phase II, open-label study evaluating the activity of imatinib in treating life-threatening malignancies known to be associated with imatinib-sensitivetyrosine kinases," Clinical Cancer Research, vol. 14, no. 9, pp. 2717-2725, 2008.

[5] M. E. Weinblatt, A. Kavanaugh, M. C. Genovese, T. K. Musser, E. B. Grossbard, and D. B. Magilavy, "An oral spleen tyrosine kinase (Syk) inhibitor for rheumatoid arthritis," The New England Journal of Medicine, vol. 363, no. 14, pp. 1303-1312, 2010.

[6] E. Scott, E. Hexner, A. Perl, and M. Carroll, "Targeted signal transduction therapies in myeloid malignancies," Current Oncology Reports, vol. 12, no. 6, pp. 358-365, 2010.

[7] H. Grant, X. Jiang, J. Stebbing et al., "Analysis of BCRABL1 tyrosine kinase domain mutational spectra in primitive chronic myeloid leukemia cells suggests a unique mutator phenotype," Leukemia, vol. 24, no. 10, pp. 1817-1821, 2010.

[8] O. Abdel-Wahab, A. Mullally, C. Hedvat et al., "Genetic characterization of TET1, TET2, and TET3 alterations in myeloid malignancies," Blood, vol. 114, no. 1, pp. 144-147, 2009.

[9] A. J. Mead, D. C. Linch, R. K. Hills, K. Wheatley, A. K. Burnett, and R. E. Gale, "FLT3 tyrosine kinase domain mutations are biologically distinct from and have a significantly more favorable prognosis than FLT3 internal tandem duplications in patients with acute myeloid leukemia," Blood, vol. 110, no. 4, pp. 1262-1270, 2007.

[10] M. M. Patnaik, R. A. Knudson, N. Gangat et al., "Chromosome 9p24 abnormalities: prevalence, description of novel JAK2 translocations, JAK2V617F mutation analysis and clinicopathologic correlates," European Journal of Haematology, vol. 84, no. 6, pp. 518-524, 2010.

[11] C. Walz, A. Chase, C. Schoch et al., "The t(8;17) (p11;q23) in the 8 p11 myeloproliferative syndrome fuses MYO18A to FGFR1," Leukemia, vol. 19, no. 6, pp. 1005-1009, 2005.

[12] A. de Klein, A. G. van Kessel, G. Grosveld et al., "A celllular oncogene is translocated to the Philadelphia chromosome in chronic myelocytic leukaemia," Nature, vol. 300, no. 5894, pp. 765-767, 1982.

[13] C. James, V. Ugo, J. P. Le Couédic et al., "A unique clonal JAK2 mutation leading to constitutive signalling causes polycythaemia vera," Nature, vol. 434, no. 7037, pp. 1144-1148, 2005.

[14] R. L. Levine, M. Wadleigh, J. Cools et al., "Activating mutation in the tyrosine kinase JAK2 in polycythemia vera, essential thrombocythemia, and myeloid metaplasia with myelofibrosis," Cancer Cell, vol. 7, no. 4, pp. 387-397, 2005.

[15] J. De Vos, M. Jourdan, K. Tarte, C. Jasmin, and B. Klein, "JAK2 tyrosine kinase inhibitor tyrphostin AG490 downregulates the mitogen-activated protein kinase (MAPK) and signal transducer and activator of transcription (STAT) pathways and induces apoptosis in myeloma cells," British Journal of Haematology, vol. 109, no. 4, pp. 823-828, 2000.

[16] S. Oku, K. Takenaka, T. Kuriyama et al., "JAK2V617F uses distinct signalling pathways to induce cell proliferation and neutrophil activation: research paper," British Journal of Haematology, vol. 150, no. 3, pp. 334-344, 2010.
[17] M. Boissinot, C. Cleyrat, M. Vilaine, Y. Jacques, I. Corre, and S. Hermouet, "Anti-inflammatory cytokines hepatocyte growth factor and interleukin-11 are over-expressed in Polycythemia vera and contribute to the growth of clonal erythroblasts independently of JAK2V617F," Oncogene, vol. 30, no. 8, pp. 990$1001,2011$.

[18] E. Parganas, D. Wang, D. Stravopodis et al., "JAK2 is essential for signaling through a variety of cytokine receptors," Cell, vol. 93, no. 3, pp. 385-395, 1998.

[19] L. M. Scott, W. Tong, R. L. Levine et al., "JAK2 exon 12 mutations in polycythemia vera and idiopathic erythrocytosis," The New England Journal of Medicine, vol. 356, no. 5, pp. 459-468, 2007.

[20] S. T. Oh, E. F. Simonds, C. Jones et al., "Novel mutations in the inhibitory adaptor protein LNK drive JAK-STAT signaling in patients with myeloproliferative neoplasms," Blood, vol. 116, no. 6, pp. 988-992, 2010.

[21] J. A. Kennedy, F. Barabé, B. J. Patterson et al., "Expression of TEL-JAKZ in primary human hematopoietic cells drives erythropoietin-independent erythropoiesis and induces myelofibrosis in vivo," Proceedings of the National Academy of Sciences of the United States of America, vol. 103, no. 45, pp. 16930-16935, 2006.

[22] P. A. Beer, P. J. Campbell, L. M. Scott et al., "MPL mutations in myeloproliferative disorders: analysis of the PT-1 cohort," Blood, vol. 112, no. 1, pp. 141-149, 2008.

[23] A. Tefferi, "Novel mutations and their functional and clinical relevance in myeloproliferative neoplasms: JAK2, MPL, TET2, ASXL1, CBL, IDH and IKZF1.," Leukemia, vol. 24, no. 6, pp. 1128-1138, 2010.

[24] L. Millecker, P. A. Lennon, S. Verstovsek et al., "Distinct patterns of cytogenetic and clinical progression in chronic myeloproliferative neoplasms with or without JAK2 or MPL mutations," Cancer Genetics and Cytogenetics, vol. 197, no. 1, pp. $1-7,2010$.

[25] M. A. Dawson, A. J. Bannister, B. Göttgens et al., "JAK2 phosphorylates histone H3Y41 and excludes HP1 $\alpha$ from chromatin," Nature, vol. 461, no. 7265, pp. 819-822, 2009.

[26] Y. Wang, W. Fiskus, D. G. Chong et al., "Cotreatment with panobinostat and JAK2 inhibitor TG101209 attenuates JAK2V617F levels and signaling and exerts synergistic cytotoxic effects against human myeloproliferative neoplastic cells," Blood, vol. 114, no. 24, pp. 5024-5033, 2009.

[27] R. Baskin, A. Majumder, and P. P. Sayeski, "The recent medicinal chemistry development of JAK2 tyrosine kinase small molecule inhibitors," Current Medicinal Chemistry, vol. 17, no. 36, pp. 4551-4558, 2010.

[28] S. Verstovsek, "Therapeutic potential of JAK2 inhibitors," American Society of Hematology: Education Program, pp. 636642, 2009.

[29] S. Verstovsek, H. Kantarjian, R. A. Mesa et al., "Safety and efficacy of INCB018424, a JAK1 and JAK2 inhibitor, in myelofibrosis," The New England Journal of Medicine, vol. 363, no. 12, pp. 1117-1127, 2010.

[30] A. Quintás-Cardama, K. Vaddi, P. Liu et al., "Preclinical characterization of the selective JAK1/2 inhibitor INCB018424: therapeutic implications for the treatment of myeloproliferative neoplasms," Blood, vol. 115, no. 15, pp. 3109-3117, 2010.

[31] T. L. Lasho, A. Tefferi, J. D. Hood, S. Verstovsek, D. G. Gilliland, and A. Pardanani, "TG101348, a JAK2-selective antagonist, inhibits primary hematopoietic cells derived from myeloproliferative disorder patients with JAK2V617F, MPLW$515 \mathrm{~K}$ or JAK2 exon 12 mutations as well as mutation negative patients," Leukemia, vol. 22, no. 9, pp. 1790-1792, 2008. 
[32] G. Wernig, M. G. Kharas, R. Okabe et al., "Efficacy of TG101348 , a selective $J A K 2$ inhibitor, in treatment of a murine model of JAK2V617F-induced polycythemia vera," Cancer Cell, vol. 13, no. 4, pp. 311-320, 2008.

[33] A. Tefferi, G. Barosi, R. A. Mesa et al., "International Working Group (IWG) consensus criteria for treatment response in myelofibrosis with myeloid metaplasia, for the IWG for Myelofibrosis Research and Treatment (IWG-MRT)," Blood, vol. 108, no. 5, pp. 1497-1503, 2006.

[34] A. Pardanani, J. R. Gotlib, C. Jamieson et al., "Safety and efficacy of TG101348, a selective JAK2 inhibitor, in myelofibrosis," Journal of Clinical Oncology, vol. 29, no. 7, pp. 789-796, 2011.

[35] J. W. Tyner, T. G. Bumm, J. Deininger et al., "CYT387, a novel $J A K 2$ inhibitor, induces hematologic responses and normalizes inflammatory cytokines in murine myeloproliferative neoplasms," Blood, vol. 115, no. 25, pp. 5232-5240, 2010.

[36] E. O. Hexner, C. Serdikoff, M. Jan et al., "Lestaurtinib (CEP701 ) is a JAK2 inhibitor that suppresses JAK2/STAT5 signaling and the proliferation of primary erythroid cells from patients with myeloproliferative disorders," Blood, vol. 111, no. 12, pp. 5663-5671, 2008.

[37] F. P. S. Santos, H. M. Kantarjian, N. Jain et al., "Phase 2 study of CEP-701, an orally available JAK2 inhibitor, in patients with primary or post-polycythemia vera/essential thrombocythemia myelofibrosis," Blood, vol. 115, no. 6, pp. 1131-1136, 2010.

[38] J. M. Goldman and J. V. Melo, "Targeting the BCR-ABL tyrosine kinase in chronic myeloid leukemia," The New England Journal of Medicine, vol. 344, no. 14, pp. 1084-1086, 2001.

[39] R. A. Mesa, "Ruxolitinib, a selective JAK1 and JAK2 inhibitor for the treatment of myeloproliferative neoplasms and psoriasis," IDrugs, vol. 13, no. 6, pp. 394-403, 2010.

[40] D. J. Barnes and J. V. Melo, "Primitive, quiescent and difficult to kill: the role of non-proliferating stem cells in chronic myeloid leukemia," Cell Cycle, vol. 5, no. 24, pp. 2862-2866, 2006.

[41] A. Mullally, S. W. Lane, B. Ball et al., "Physiological JAK2V617F expression causes a lethal myeloproliferative neoplasm with differential effects on hematopoietic stem and progenitor cells," Cancer Cell, vol. 17, no. 6, pp. 584-596, 2010.

[42] M. Lu, W. Zhang, Y. Li et al., "Interferon- $\alpha$ targets JAK2V617Fpositive hematopoietic progenitor cells and acts through the p38 MAPK pathway," Experimental Hematology, vol. 38, no. 6, pp. 472-480, 2010.

[43] J.-J. Kiladjian, R. A. Mesa, and R. Hoffman, "The renaissance of interferon therapy for the treatment of myeloid malignancies," Blood, vol. 117, no. 18, pp. 4706-4715, 2011.

[44] T. Manshouri, Z. Estrov, A. Quintás-Cardama et al., "Bone marrow stroma-secreted cytokines protect JAK2V617F-mutated cells from the effects of a JAK2 inhibitor," Cancer Research, vol. 71, no. 11, pp. 3831-3840, 2011.

[45] E. Jabbour, D. Thomas, H. Kantarjian et al., "Comparison of thalidomide and lenalidomide as therapy for myelofibrosis," Blood, vol. 118, no. 4, pp. 899-902, 2011.

[46] K. H. Begna, R. A. Mesa, A. Pardanani et al., "A phase-2 trial of low-dose pomalidomide in myelofibrosis," Leukemia, vol. 25, no. 2, pp. 301-304, 2011.

[47] C. Besses, A. Álvarez-Larrán, L. Martínez-Avilés et al., "Modulation of JAK2V617F allele burden dynamics by hydroxycarbamide in polycythaemia vera and essential thrombocythaemia patients," British Journal of Haematology, vol. 152, no. 4, pp. 413-419, 2011. 


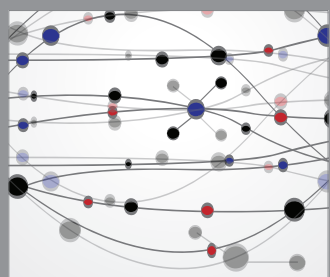

The Scientific World Journal
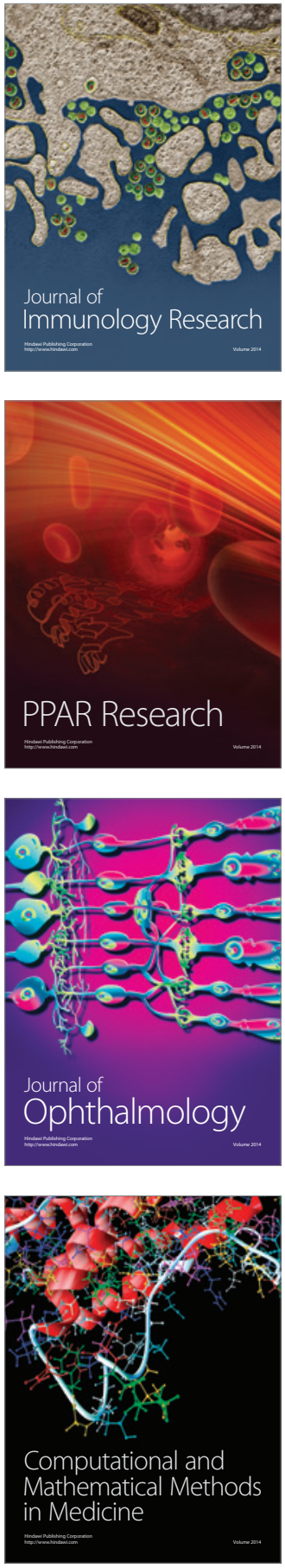

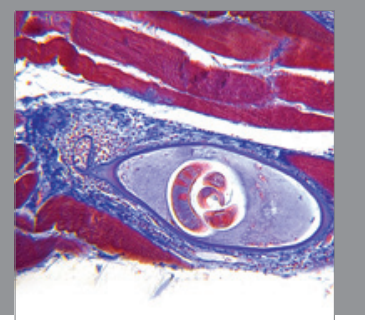

Gastroenterology

Research and Practice
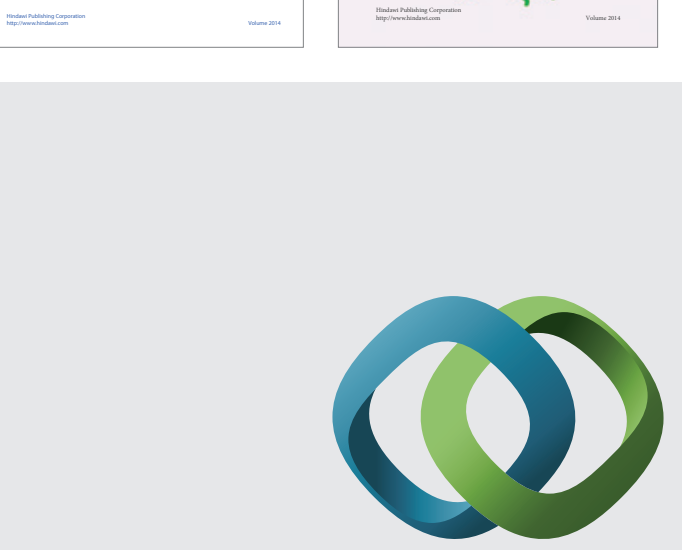

\section{Hindawi}

Submit your manuscripts at

http://www.hindawi.com
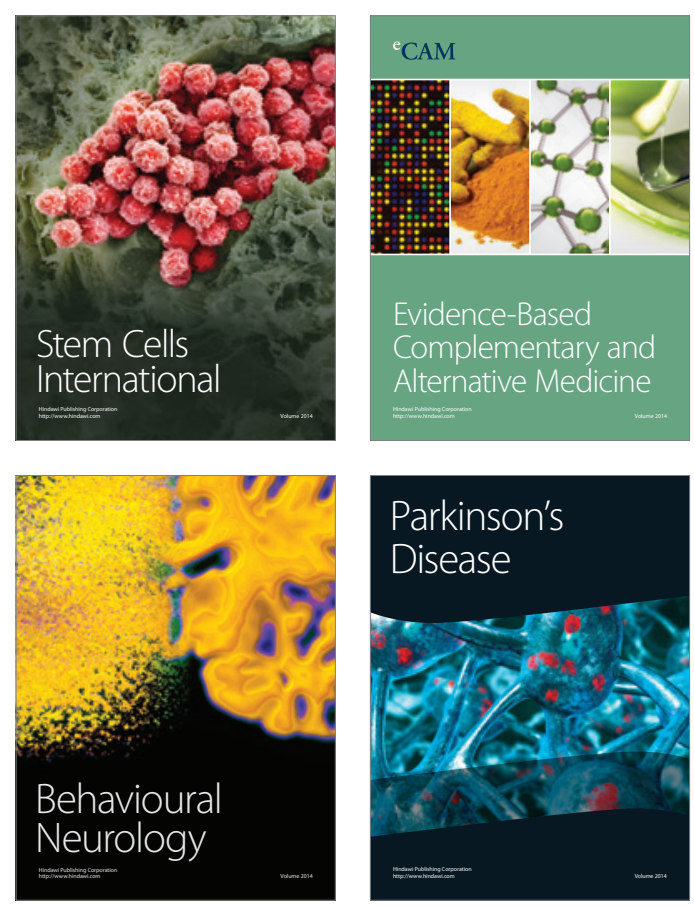

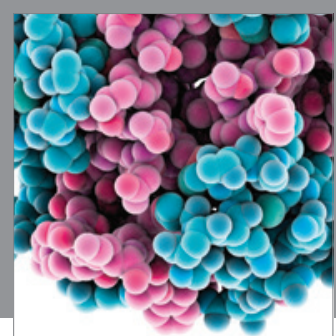

Journal of
Diabetes Research

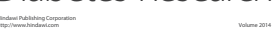

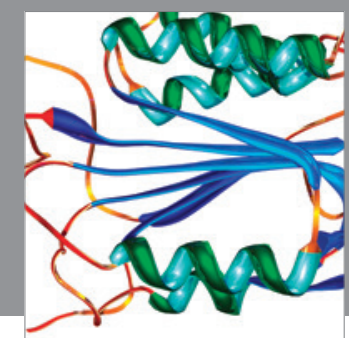

Disease Markers
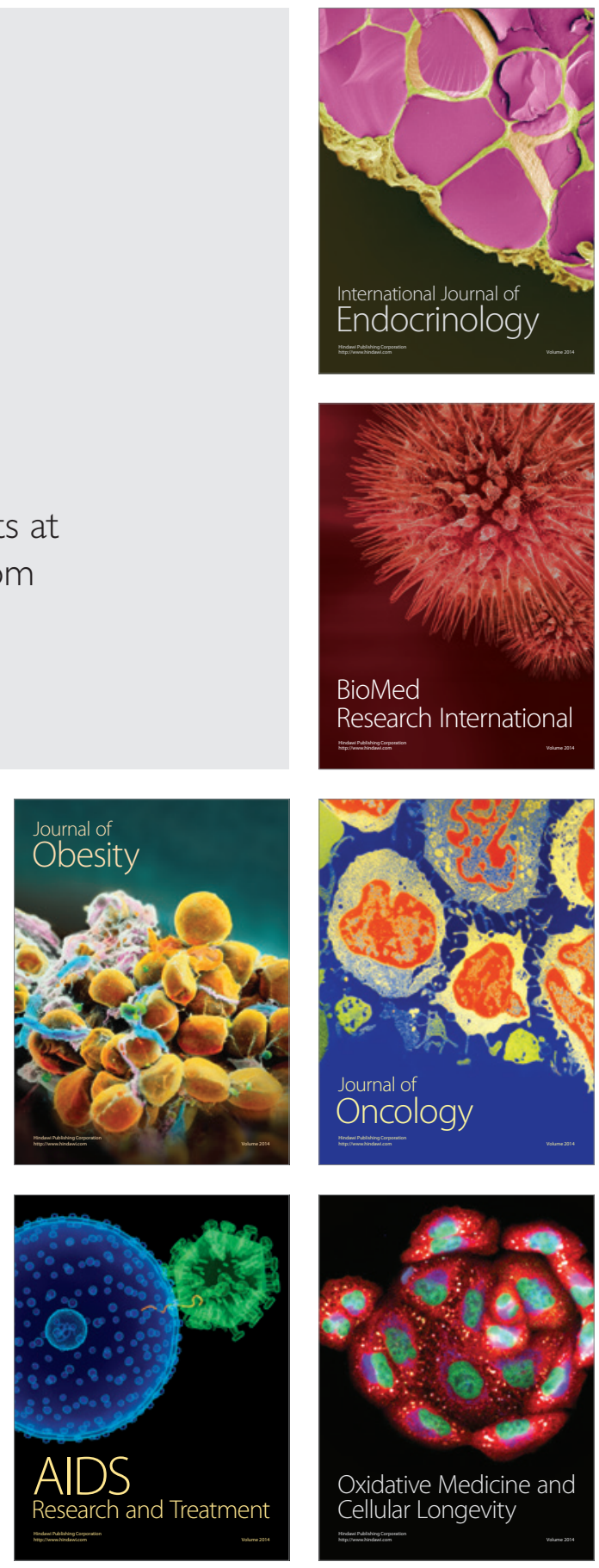